Received: 2016.12 .03

Accepted: 2017.02 .20

Published: 2017.11.17

\section{Internal Mammary Artery Anastomoses in Patients without Breast Lesions (BIRADS 1) and in Patients with Malignant Breast Lesions (BIRADS 6) on Magnetic Resonance Imaging (MRI)}

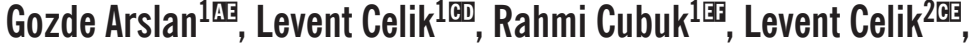 \\ Mehmet Mahir Atasoy ${ }^{1 \mathrm{CF}}$ \\ ${ }^{1}$ Department of Radiology, Maltepe University, Maltepe, Istanbul, Turkey \\ 2 Department of Radiology, Radiologica Imaging Center, Istanbul, Turkey
}

Author's address: Gozde Arslan, Department of Radiology, Maltepe University, Maltepe, Istanbul, Turkey, e-mail: gunesgozde@gmail.com

\begin{tabular}{|c|c|}
\hline Background: & $\begin{array}{l}\text { We aimed to compare the frequency of IMA (intern } \\
\text { left breasts in patients without breast lesions and }\end{array}$ \\
\hline laterial/Methods: & $\begin{array}{l}\text { A total of } 100 \text { patients with no breast lesions } \\
\text { lesions were included in the study. The anastomo } \\
\text { on MIP and post-contrast Tl-weighted magneti } \\
\text { scans were read by a radiologist. }\end{array}$ \\
\hline Results: & $\begin{array}{l}\text { In patients with no breast lesions, IMA anastom } \\
\text { with malignant breast lesions, IMA anastomo } \\
\text { with malignant lesions, ipsilateral IMA was ru } \\
\text { contralateral IMA. }\end{array}$ \\
\hline Conclusions: & $\begin{array}{l}\text { No statistically significant difference was found } \\
\text { patients with malignant breast lesions with res } \\
\text { and left breasts. The frequencies were higher tha }\end{array}$ \\
\hline MeSH Keywords: & Anastomosis, Surgical • Breast • Mammary Arteries \\
\hline PDF fil & http://www.polradiol.com/abstract/index/idArt/902 \\
\hline
\end{tabular}

\section{Background}

The internal mammary artery (IMA) and the lateral thoracic artery (LTA) are the two main arteries that supply blood to the breasts. IMA is better known than LTA, as it is used for coronary revascularization grafting [1-3]. IMA has a critical importance for the breast tissue; it is the main source of blood supply for the breasts and also for breast tumor cells that can spread through IMA to other parts of the body. In our study, we investigated the presence of anastomoses between right and left IMAs using magnetic resonance imaging (MRI). We included patients with malignant breast lesions and patients with no lesions in the breast. We aimed to investigate whether IMA anastomoses are more frequent in patients with breast malignancy than in healthy participants.

\section{Material and Methods}

This retrospective study was performed in our university hospital (Maltepe University Hospital, Radiology Department) between 2013 and 2016. A total of 100 women who had biopsy proven malignant breast lesions on MRI (BIRADS 6) and a total of 100 women who had normal breasts on MRI (BIRADS 1) were included. Women were aged between 21 and 87 years. The study was approved by our local ethics committee, and signed consent was obtained from all patients. MR images of lesions were 


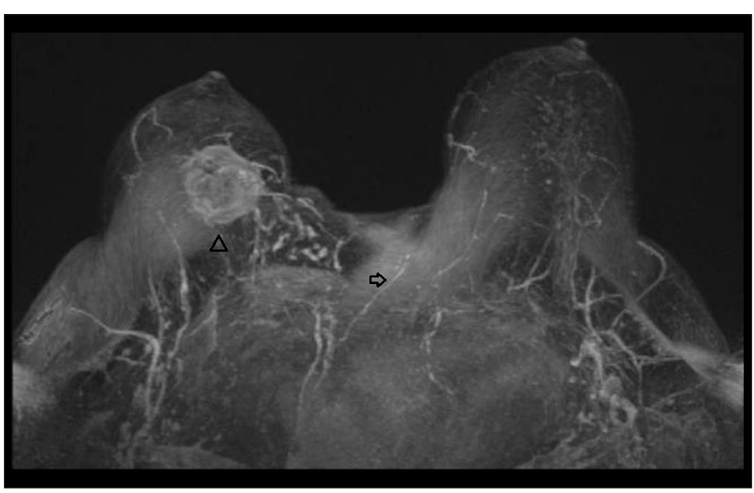

Figure 1. Axial maximum intensity projection (MIP, MRI) of the breast. A large tumor is seen in the right breast (arrowhead). The right IMA is giving a branch to the left IMA (arrow).

evaluated by a single radiologist. IMAs were mostly analyzed on post-contrast subtraction Tl-weighted sequences (TlWS). However, maximum intensity projection (MIP) images were also analyzed to check for the presence of microvascular anastomoses. Our radiologist checked IMAs from their origin (subclavian artery) to the branches inside the breasts, and looked for anastomoses between the IMAs of right and left breasts. The radiologist was blinded to the main breast lesion and to its classification (BIRADS 1 or 6). Before MRI, histopathological diagnoses of the lesions in patients with BIRADS lesions were obtained by ultrasonography-guided tru-cut breast biopsy.

MRI was performed with a 1.5-T MR scanner (Intera, Philips Medical Systems, Best, The Netherlands), with a dedicated double-breast surface coil and patients in the prone position. Tl-weighted fast gradient echo fat-suppressed sequences (TE/TR, 2.4/4.6 ms; flip angle, $10^{\circ}$; FOV, $360 \times 360 \mathrm{~mm}^{2}$, acquired voxel size, $0.9 \times 0.9 \times 2.5 \mathrm{~mm}^{3}$; reconstructed voxel size, $0.83 \times 0.83 \times 2.50 \mathrm{~mm}^{3}$; total scanning, time $60 \mathrm{~s}$ ) were acquired in the axial plane before contrast agent administration and $0,1,2,3,4,5$, and $7 \mathrm{~min}$ after administration of contrast agent $(0.1 \mathrm{mmol} / \mathrm{kg}$ of gadolinium diethylenetriamine pentaacetic acid (Gd-DTPA)).

Also, T2-weighted fat-suppressed spin echo sequences were acquired in the axial plane before contrast agent (TE/TR, 110/7548 ms; inversion delay SPAIR, $80 \mathrm{~ms}$; flip angle $90^{\circ}$; FOV, $380 \times 380 \mathrm{~mm}^{2}$; acquired voxel size, $1.06 \times 1.74 \times 3.0 \mathrm{~mm}^{3}$; reconstructed voxel size, $0.94 \times 0.94 \times 3.00 \mathrm{~mm}^{3}$; total scanning time, $242 \mathrm{~s}$ ).

Exclusion criteria

- Prior history of breast cancer and prior history of chemotherapy/radiotherapy on the breast or chest wall area.

- Prior history of breast surgery (breast-conserving/radical mastectomy, breast augmentation etc.)

- Prior history of coronary bypass surgery

- Bilateral breast tumors

\section{Statistical Analysis}

We used the NCSS software (Number Cruncher Statistical System, 2007, Kaysville, Utah, USA) for statistical analysis. The Pearson's chi-squared test was used for comparison of qualitative variables along with descriptive statistical

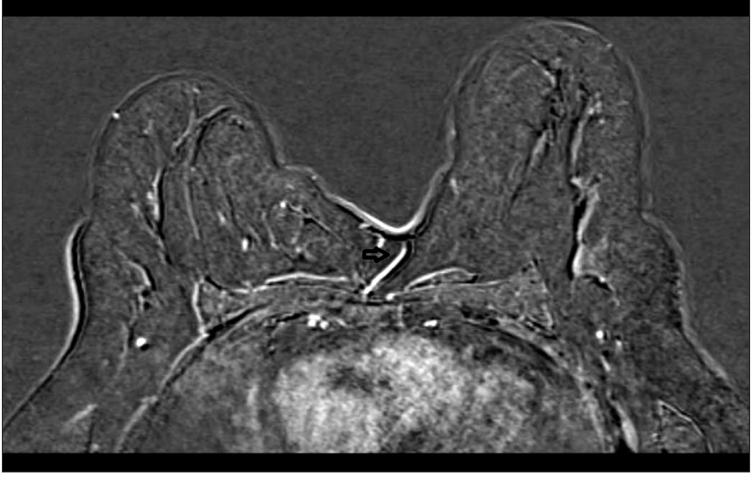

Figure 2. Axial post-contrast T1WS subtraction MRI of the same patient as in Figure 1. The right IMA is giving a branch to the left IMA (arrow).

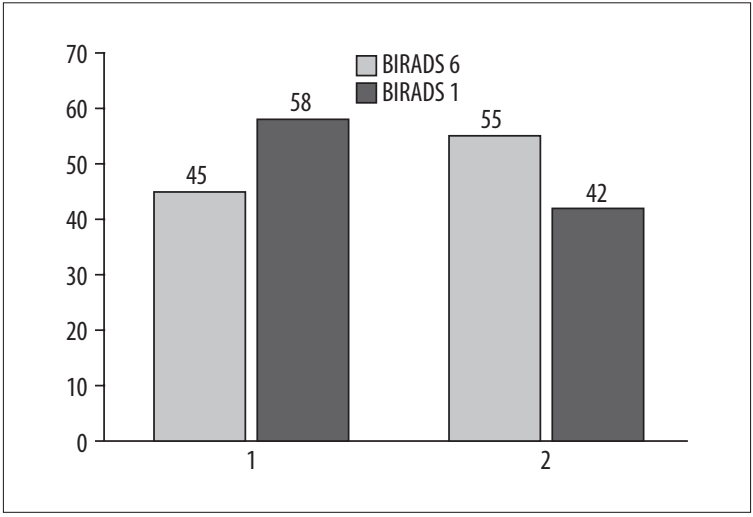

Figure 3. Distribution of anastomoses with respect to the presence of a lesion. 1 - group with anastomoses, 2 - group without anastomoses.

methods (frequency, ratio). The accepted limit of significance was $\mathrm{p}<0.05$.

\section{Results}

A total of 200 female patients who underwent breast MRI in the Radiology Department of the Maltepe University Hospital between the years 2013 and 2016 were enrolled in the study. Their mean age was 51 years (21-87 years).

One half of the patients $(n=100)$ were category BI-RADS l, and they had no mammary lesions. In 45 of these patients, IMA, which was examined from its point of origin until the branching point, anastomosed with the contralateral IMA. The remaining half of patients $(n=100)$ were category BI-RADS 6, and they had malignant breast lesions confirmed by histopathology. In 58 of these patients, IMA anastomosed with the contralateral IMA (Figures 1, 2).

Although there was no statistically significant difference between the two groups with respect to the frequency of anastomoses $(p=0.066 ; p>0.05)$, patients with malignant lesions had a higher frequency of anastomoses (Figure 3).

\section{Discussion}

An anastomosis is a connection between blood vessels. It might be congenital or acquired. There are many natural 
anastomoses in the human body. One of the most wellknown example is the circle of Willis. It provides communication between the blood supply of the forebrain and hindbrain, which is essential for collateral blood flow in cases of internal carotid artery (ICA) stenosis or occlusion. Such natural anastomoses can therefore be lifesaving. Not all vascular structures are the same in every human being, and variabilities in vascular connections are commonly observed. One of these variations is seen in IMA, which is the main blood supplier of the breast. The breast is supplied mainly by two arteries. The most important of these vascular structures is IMA - a branch of the internal thoracic artery, which enters the breast medially from both sides of the sternum. The lateral thoracic artery - a branch of the axillary artery, enters the breast through the outer half. IMAs of both breasts might be connected by an anastomosis, either congenital or acquired.

The presence of benign or malignant tumor cells in the breast might stimulate the proliferation of the vascular plexus on the same side in order to increase blood supply. This process is regulated by some angiogenic activators $[4,5]$. If these substances and vascular support are not available in sufficient amounts, tumor cells might become necrotic or apoptotic [6,7]. Development of an anastomosis within the vascular plexus might further increase blood supply and stimulate proliferation.

In our study, we evaluated IMAs in patients with malignant breast tumors and in patients with no breast lesions. To our knowledge, there has been no other study focusing on this topic. The frequencies of IMA anastomoses were not significantly different between patients with and without malignant breast lesions. This means that IMA of both breasts might have congenital or acquired anastomoses that are not related to breast lesions. Detecting these anastomoses is significant with respect to interventional therapies and other treatment modalities. This phenomenon might also explain contralateral breast metastases that are observed in $10 \%$ to $15 \%$ of patients 15 years after treatment and even the higher proportion of longer-term survivors [8]. Studies have demonstrated that contralateral breast cancer is the most common new primary cancer in breast cancer patients, accounting for $30-50 \%$ of all new primary cancers [9-11]. Whether these cancers are primary tumors or are metastases of the existing breast cancer remains unclear. In a genome study, Alkner et al. confirmed the presence of metastases to the contralateral breast by showing common clonal origin [12]. Similar to lung cancer, metastases to the contralateral breast are considered as distant metastases. Due to that, breast cancer survivors with tumors in the contralateral breast are currently treated, in addition to unilateral mastectomy, with prophylactic hormone therapy and radiotherapy [13] or contralateral mastectomy. These therapies have favorable short-term and long-term effects. Our study demonstrated a high incidence of interconnections between the vascular structures of both breasts. In the future, obliteration of these vascular structures might be planned in high-risk patients with BRCAl and BRCA2 mutations.

Furthermore, the high incidence of vascular anastomoses in both breasts raises questions about staging of contralateral breast metastases that are currently considered as distant organ metastases. Theoretically, tumors in the inner breast quadrant might spread to the contralateral breast through IMA anastomoses much easier than to the outer breast quadrant of the ipsilateral breast. In view of the above-mentioned information and findings, awareness of vascular anastomoses between both breasts seems important. Preventing the development of such anastomoses by obliteration of vascular plexuses might hinder metastatic spread.

A small study sample and lack of evaluation of the BRCA1 and BRCA2 mutations might be considered as limitations of our study.

\section{Conclusions}

In our study, we observed that the frequency of IMA anastomoses in right and left breasts was high in both patient groups (patients with no breast lesions and patients with malignant breast tumors). The high incidence of these anastomoses provides insight into the mechanisms of metastatic spread to the contralateral breast. Moreover, future therapies might target such anastomoses. We believe that a better understanding of vascular anastomoses in the breast will play an important role in the future. To this end, further studies with more advanced methods are needed.

\section{Conflict of interest}

The authors have no conflict of interest.

\section{References:}

1. Vineberg AM: Development of the anastomoses between the coronary vessel and a transplanted internal mammary artery. Can Med Assoc J, 1946; 55: 117

2. Kolessov VL: Mammary artery-coronary artery anastomosis as method of treatment for angina pectoris. J Thoracic Cardiovasc Surg, 1967; 54: 535-44

3. Loop FD, Lytle BW, Cosgrove DM et al: Influence of the internalmammary-artery graft on 10-year survival and other cardiac events. N Engl J Med, 1986; 314: 1-6

4. Folkman $\mathrm{J}$ : What is the evidence that tumors are angiogenesis dependent?. J Natl Cancer Inst, 1990; 82(1): 4-6

5. Salven P, Lymboussaki A, Heikkilä P et al: Vascular endothelial growth factors VEGF-B and VEGF-C are expressed in human tumors. Am J Pathol, 1998; 153(1): 103-8
6. Nishida N, Yano H, Nishida $\mathrm{T}$ et al: Angiogenesis in cancer. Vasc Health Risk Manag, 2006; 2(3): 213-19

7. Holmgren L, O'Reilly MS, Folkman J: Dormancy of micrometastases: Balanced proliferation and apoptosis in the presence of angiogenesis suppression. Nat Med, 1995; 1(2): 149-53

8. Freedman GM, Anderson PR, Hanlon AL et al: Pattern of local recurrence after conservative surgery and whole-breast irradiation. Int J Radiat Oncol Biol Phys, 2005; 61(5): 1328-36

9. Gao X, Fisher SG, Emami B: Risk of second primary cancer in the contralateral breast in women treated for early-stage breast cancer: A population-based study. Int J Radiat Oncol Biol Phys, 2003; 56(4): 1038-45

10. Harvey EB, Brinton LA: Second cancer following cancer of the breast in Connecticut, 1935-1982. Natl Cancer Inst Monogr, 1985; 68: $99-112$ 
11. Schaapveld M, Visser O, Louwman WJ et al: The impact of adjuvant therapy on contralateral breast cancer risk and the prognostic significance of contralateral breast cancer: A population based study in the Netherlands. Breast Cancer Res Treat, 2008; 110(1): 189-97
12. Alkner S, Tang MH, Brueffer $\mathrm{C}$ et al: Contralateral breast cancer can represent a metastatic spread of the first primary tumor: Determination of clonal relationship between contralateral breast cancers using next-generation whole genome sequencing. Breast Cancer Res, 2015; 17: 102

13. Brenner DJ, Shuryak I, Russo S, Sachs RK: Reducing second breast cancers: A potential role for prophylactic mammary irradiation. $J$ Clin Oncol, 2007; 25(31): 4868-72 